\title{
Three-Dimensional Forward Modeling for TEM by Vector Finite Element Method
}

\author{
Jia Li, Xiu Li \\ Geology Engineering and Geometrics, Chang' an University, \\ Xi'an, China
}

\begin{abstract}
Transient electromagnetic method has gradually became a popular geophysical prospecting method in recent years in China and rest of the world. Considering the complexity of the transient electromagnetic theory, the theory of technology is still imperfect, especially in the area of $3 \mathrm{D}$ inversion processing and interpretation, which is far from practical level. With this situation, this paper studies the three-dimensional forward modeling for transient electromagnetic method by vector finite element method. And we use quadratic field method as a way to load source, then the frequency domain of three-dimensional forward modeling is realized. In the process, the sparse matrix is stored by the CSR format and the equations are solved by the parallel solver PARDISO in the MKL library. Then we transform the solution form frequency domain to time domain. We discrete frequency by the logarithmic interval. And we obtain the frequency domain response that we need by using cubic spline function, and then realize the transformation of time domain by cosine transform. By comparing the results of typical layered model analysis, the validity of the vector finite element method is proved. The three-dimensional model test shows the accuracy of the vector finite element method. It can objectively reflect the scale and the position of the underground abnormal body.
\end{abstract}

Keywords-transient electromagnetic method; vector finite element method; three-dimensional forward modeling

\section{INTRODUCTION}

Considering the complexity of the transient electromagnetic theory, the theory is still imperfect, especially in the area of $3 \mathrm{D}$ inversion processing and interpretation, which is far from practical level. In recent years, many geophysical scholars devoted to the 3D forward modeling of transient electromagnetic, and the main methods include the finite difference time domain method, finite element method, finite volume method, the volumetric integral equation method, etc. But because of the complexity of the finite element theory and the higher demand of the software to solve the large equations, it is few to solve the TEM field by the three dimensional finite element method. However, considering the characteristics of finite element method, such as the accurate simulation of the complex structure model, the 3D electromagnetic field simulation of the model with terrain, etc., and with the improvement of computer hardware, it is necessary to study the three dimensional numerical simulation of TEM field by finite element method, so as to study the propagation rule and response characteristics of the TEM filed in a complex geoelectric model in order to lay the foundation for three-dimensional interpretation technology. Threedimensional forward modeling by finite element method

\author{
Weihua Yao \\ China Coal Technology \& Engineering Group XI'AN \\ Research Institute, Xi'an, China
}

includes the nodal finite element method and the vector finite element method. As different mediums have the different electrical and magnetic parameters, it is hard to deal with the different boundary conditions of the medium interface. Therefore it creates the so-called ' pseudo solution ' phenomenon when using the nodal finite element. The nodal Finite element method gives freedom to cell node, while the vector finite element method uses vector basis functions to approximate unknown functions, and gives freedom to unit grid edge. Because vector basis functions have a constant value on the edge and the direction along the edge direction, the vector finite element method not only ensures the continuity of the tangential component of electromagnetic field, but also does not impose the continuity of the Normal component of electromagnetic field. So the vector finite element method can effectively avoid pseudo solution of nodal finite element method.

\section{THEORY AND METHOD}

In this paper, we starting from the Maxwell's equations of frequency domain, and using the Dirichlet boundary conditions.

$$
\begin{aligned}
& \nabla \times \vec{E}=i \omega \mu \vec{H} \\
& \nabla \times \vec{H}=\sigma \vec{E}+\vec{J}_{s} \\
& \nabla \cdot \vec{E}=0 \\
& \nabla \cdot \vec{H}=0
\end{aligned}
$$

And the $\vec{E}$ is Electric field intensity, $\vec{H}$ is magnetic field intensity, $\vec{j}_{s}$ is impressed current source term, $\sigma 、 \mu$ respectively is the conductivity, permeability, $\omega$ is the angular frequency.

We use quadratic field method as a way to load source, that is:

$$
\vec{E}=\vec{E}^{a}+\vec{E}^{b}
$$

And $\vec{E}^{b}$ is the background field, $\vec{E}^{a}$ is the anomalous field.

Then we can get the twin twist degree equation for the electric field:

$$
\nabla \times \nabla \times \vec{E}^{a}-i \omega \mu \sigma \vec{E}^{a}=i \omega \mu \sigma^{a} \vec{E}^{b}
$$

Among them $\sigma^{a}=\sigma-\sigma^{b}$.

Then can get the variation equation of electric field:

$$
\int_{V_{e}} f \cdot\left(\nabla \times \nabla \times \vec{E}^{a}-i \omega \mu_{0} \sigma^{a} \vec{E}^{a}-i \omega \mu_{0} \sigma^{a} \vec{E}^{b}\right) d e=0
$$


Reduction to:

$$
\int_{V}\left(\nabla \times \vec{E}^{a}\right) \cdot(\nabla \times \vec{f})-i \omega \sigma \vec{E}_{0} \vec{E}^{a} \cdot \vec{f}-i \omega \sigma^{a} \mu_{0} \vec{E}^{b} \cdot \vec{f} d V=0
$$

And the $\mathrm{f}$ is the vector basis functions.

It's written in matrix form: $A \cdot E=B$

Among them

$$
\begin{aligned}
& A_{e}=\iiint_{V e}\left[\left(\nabla \times N_{e}^{i}\right) \cdot\left(\nabla \times N_{e}^{j}\right)-i \omega \mu_{0} \sigma N_{e}^{i} \cdot N_{e}^{j}\right] d e \\
& B_{e}=i \omega \mu_{0} \sigma \iiint_{V e} \vec{E}_{e}^{b} \cdot N_{e}^{i} d e
\end{aligned}
$$

$E_{e}$ is the column vector that is formed with the projection values that the anomalous field in the rectangular block unit e is on the side of the edge, $\vec{E}^{b}$ is the column vector that is formed with the projection values that the background field in the rectangular block unit e is on the side of the edge. After the element analysis of all the grid, then generate the stiffness matrix by the overall synthesis of the units. Then we can get large sparse linear equations. There are a number of storage methods of the stiffness matrix, such as half the bandwidth storage, Coordinate store, etc. In this paper, the sparse matrix is stored by the CSR format and the equations are solved by the parallel solver PARDISO in the MKL library.

\section{THE ALGORITHM VALIDATION AND MODEL CALCULATION}

In order to verify the accuracy of the algorithm, we use the $\mathrm{H}$ model The size of the square loop source is $100 \mathrm{~m} \times$ $100 \mathrm{~m}$, the current is $10 \mathrm{~A}$, the size of the subdivision is $40000 \mathrm{~m} \times 40000 \mathrm{~m} \times 40000 \mathrm{~m}$, the number of grid is $63 \times 63 \times 53$, the smallest size of mesh subdivision is $10 \mathrm{~m}$, the source is inside the uniform grid, and source center is located in the origin of coordinates $(0,0,0)$, the geoelectric parameters of $\mathrm{H}$ model are set in the following TABLE I. THE GEOELECTRIC PARAMETERS OF H MODEL.

TABLE I. THE GEOELECTRIC PARAMETERS OF H MODEL

\begin{tabular}{cccccc}
\hline $\begin{array}{c}\text { Geoelectric } \\
\text { model }\end{array}$ & $\begin{array}{c}\text { The } \\
\text { resistivity } \\
\text { of first } \\
\text { layer }\end{array}$ & $\begin{array}{c}\text { The } \\
\text { resistivity } \\
\text { of second } \\
\text { layer }\end{array}$ & $\begin{array}{c}\text { The } \\
\text { resistivity } \\
\text { of third } \\
\text { laye }\end{array}$ & $\begin{array}{c}\text { thickness } \\
\text { of first } \\
\text { layer }\end{array}$ & $\begin{array}{c}\text { thickness } \\
\text { of } \\
\text { second } \\
\text { layer }\end{array}$ \\
\hline H model & $100 \Omega \cdot \mathrm{m}$ & $1 \Omega \cdot \mathrm{m}$ & $100 \Omega \cdot \mathrm{m}$ & $80 \mathrm{~m}$ & $50 \mathrm{~m}$
\end{tabular}

The coordinates of measuring point are $(20,20,0)$,it can be seen from the fig. 1 that the vertical $\mathrm{Z}$ component response curve of $\mathrm{H}$ model of the vector finite element method and the response of digital filtering method fit better, the error of every moment is small, and keeps within 5\%, which shows the accuracy of the method in this paper.

Then we design a 3D model to calculate. In homogeneous half space put a tilted low resistance body, and put a electrical source with $110 \mathrm{~m}$ length on the ground as excitation source, then receive response of TEM field in the air, the resistivity of the homogeneous half space is $100 \Omega \cdot m$, the resistivity of the tilted low resistance body is $10 \Omega \cdot m$, the flight altitude of unmanned aerial vehicle is $80 \mathrm{~m}$, the emission current is $10 \mathrm{~A}$, the size of the subdivision is $40000 \mathrm{~m}$ $\times 40000 \mathrm{~m} \times 40000 \mathrm{~m}$, the number of grid is $63 \times 63 \times 53$, the smallest size of mesh subdivision is $10 \mathrm{~m}$, the source is inside the uniform grid, and source center is located in the origin of coordinates $(0,0,0)$, the model parameters as shown in fig. 2 and fig. 3 .

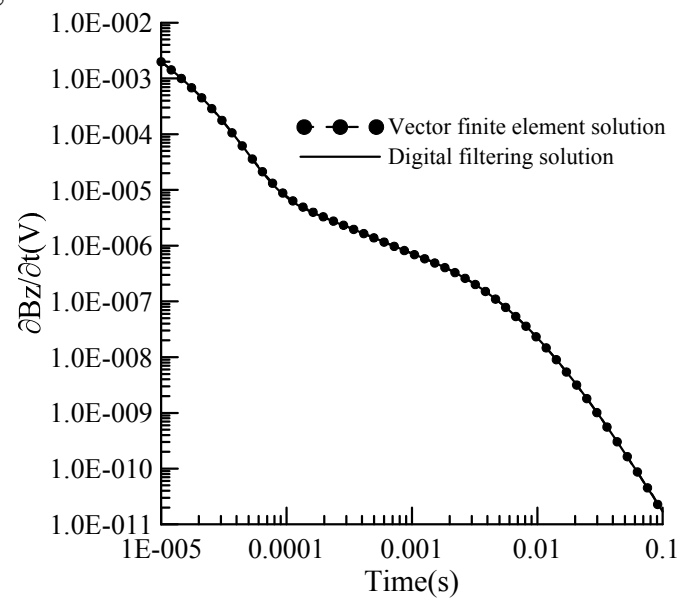

(a)

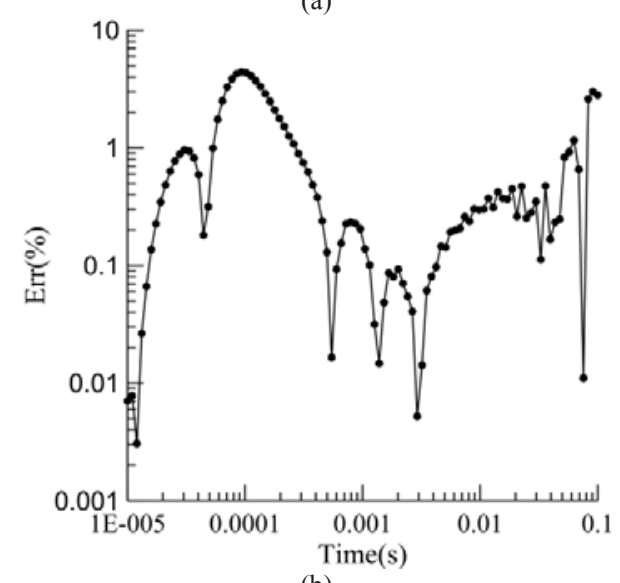

(b)

Fig.1. Test of vector finite element method with the $\mathrm{H}$ model(a)Comparison between results of vector finite element method and those of digital filtering method of vertical $\mathrm{Z}$ component attenuation response of $\mathrm{H}$ model (b) Relative error of forward modeling results of $\mathrm{H}$ model

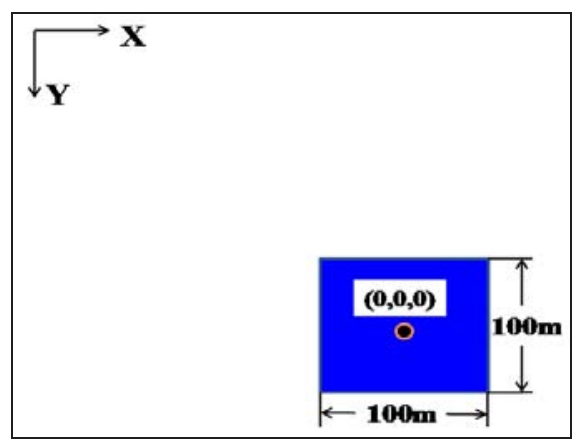

Fig.2. The model vertical view of $\mathrm{Z}$ direction 


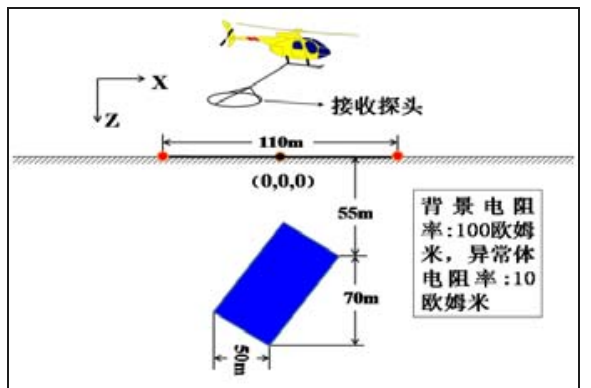

Fig.3. The model vertical view of Y direction

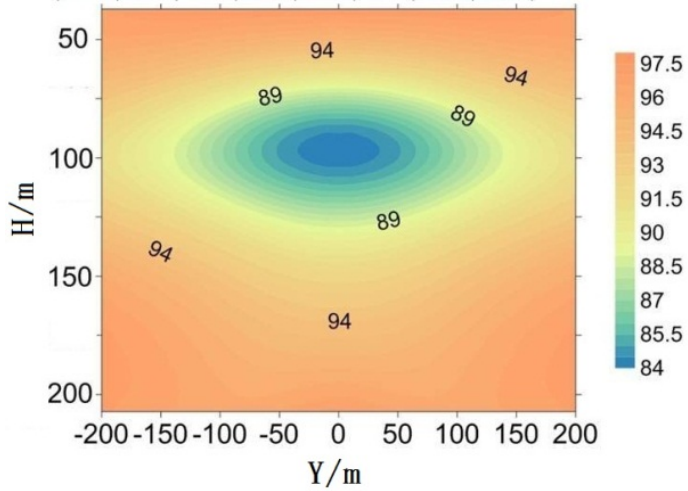

(a) $x=10 m$

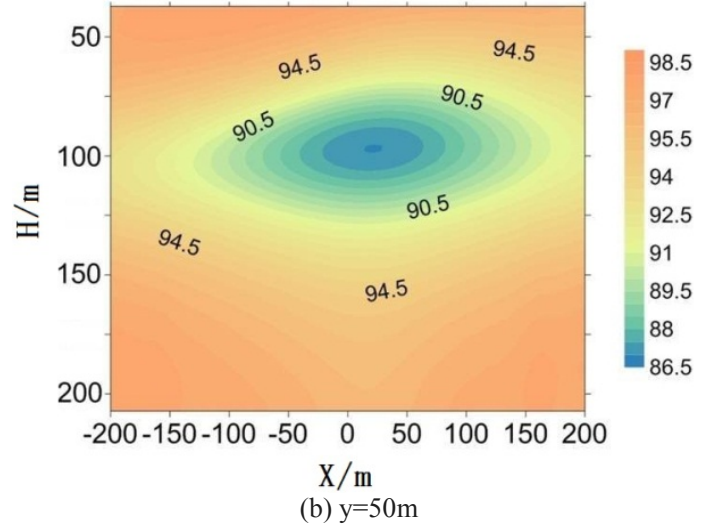

Fig.4. Multi-component full field apparent resistivity of flight altitude $80 \mathrm{~m}$
We get the transient electromagnetic response at $X=10 \mathrm{~m}$ and $\mathrm{Y}=50 \mathrm{~m}$ by the vector finite element method. As is shown in fig.4.

Apparent resistivity profile can clearly reflect the low resistivity anomalies, and it is consistent with the design model, which further validates the reliability of threedimensional forward modeling for transient electromagnetic by vector finite element method.

\section{CONCLUSIONS}

We use the three layer model to verify the results accuracy of the algorithm, and calculate the air model in the case of electrical source, design a 3D model to calculate and get the clearly result. Therefore, we suggest that that threedimensional forward modeling for transient electromagnetic by vector finite element method is reliable.

\section{ACKNOWLEDGMENT}

This research is funded by the National Natural Science Foundation of China under the grants (51139004)

\section{REFERENCES}

[1] LI Jian-hui, ZHU Zi-qiang, ZENG Si-hong, et al,2012, Progress of forward computation in transient electromagnetic method. Progress in Geophys. (in Chinese), 27(4),1393-1400. 